\title{
A modular integration and multiresolution framework for image interpretation
}

\author{
K SUNIL KUMAR and U B DESAI \\ Signal Processing and Artificial Neural Networks Laboratory, Department \\ of Electrical Engineering, Indian Institute of Technology - Bombay, Powai, \\ Mumbai 400076, India \\ e-mail: ubdesai@ee.iitb.ernet.in
}

MS received 19 May 1997; revised 22 December 1997

\begin{abstract}
In this paper, we give a generalized formulation for a vision problem in the framework of modular integration and multiresolution. The developed framework is used to solve the high-level vision problem of scene interpretation. The formulation essentially involves the concept of reductionism and multiresolution, where the given vision task $\mathcal{V}$ is broken down into simpler subtasks $\mathcal{V}_{1}, \mathcal{V}_{2}, \ldots, \mathcal{V}_{m}$. Moreover, instead of solving the vision task $\mathcal{V}^{\Omega}=\mathcal{V}$ at the finest resolution $\Omega$, we solve the synergetically coupled vision subtasks at coarser resolutions $\nu^{\Omega-N}$ for $\Omega \geq N>0$ and use the results obtained at resolution $(\Omega-N)$ to solve $\mathcal{V}^{\Omega-N+1}$, the vision task at resolution $(\Omega-N+1)$. Image interpretation is a two-phased analysis problem where some salient features or objects in an image are identified by segmenting the image and the objects in the segmented image are interpreted based on their spatial relationships. We present a solution to the joint segmentation and interpretation problem in the proposed generalized framework. For the interpretation part we exploit the Markov Random Field (MRF) based image interpretation scheme developed by Modestino and Zhang. Experimental results on both indoor and outdoor images are presented to validate the proposed framework.
\end{abstract}

Keywords. Modular integration; multiresolution framework; image interpretation; Markov random field.

\section{Introduction}

Interpretation is a high-level description of the environment from which the image was taken. It is essentially an analysis problem where we try to understand the image by segmenting ${ }^{1}$ and subsequently identifying some important features or objects and analysing

\footnotetext{
${ }^{1}$ Segmentation is a process of dividing the image into regions having similar properties
} 
them depending on their spatial relationship. Image interpretation is a hard task to automate, considering the fact that the human visual system, undoubtedly the best visual system, is fooled quite often, especially when other sources of information which help in disambiguating illusions are hidden.

Image interpretation is knowledge-based processing, which requires the use of both lowlevel processing (image processing techniques of contrast enhancement, computer vision techniques of segmentation, feature extraction, region labeling) and high-level vision tasks involving processing a great amount of non-image related knowledge underlying the scene representation, for example, knowledge about the world physical constraints influencing entities and their environment (Schalkoff 1989). At low levels, the basic processing unit being pixel, there is no simple computational transformation that will map arrays of pixels onto stored symbolic concepts represented in the high-level knowledge-base. It is generally accepted that many stages of processing must take place for reliable interpretation of a scene. For high-level interpretation, the principal unit of information is a symbolic description of an object, or a set of image events, sometimes referred to as symbolic tokens, extracted from the image. The description includes relationships to other $2 \mathrm{D}$ symbolic tokens extracted from the image, such as lines, segments and other objects in the 3D scene being viewed. It also includes pointers to elements of general knowledge that have been used to support the interpretation process.

Image interpretation has been under investigation for about a couple of decades and consequently the literature is quite vast. Early work on image interpretation was based on image features which were dealt with in isolation. These features were classified into a finite set of classes, namely, interpretation labels; presumably this scheme is not robust especially when the low-level vision tasks give erroneous outputs. More recent approaches adopt knowledge-based systems for image interpretation. Here, a great amount of nonimage related knowledge underlying the scene representation is used along with the spatial constraints. Thus, there is a possibility of even an ambiguous object being recognised, based on the successful recognition of its neighbourhood objects.

The early work in knowledge-based image interpretation is summarized by Nagao \& Matsuyama (1980), Binford (1982), and Ohta (1985), and more recently by Chu \& Aggarwal (1992). Rule-based strategies are especially appropriate in view of lack of complete models and algorithmic strategies (Puliti \& Tascini 1993). Markov Random Field (MRF) models are being used for image interpretation with a view to make the interpretation systematic and domain independent (Modestino \& Zhang 1992; Kim \& Yang 1993, 1995; Kumar \& Desai 1996). Most of the interpretation schemes assume the availability of a good segmented image of the scene $a$ priori. But in practice, obtaining a good segmented image is difficult for the simple reason that segmentation itself depends on, and hence is a function of, the output of interpretation. In this paper, we propose a modular integration and multiresolution framework for the high-level vision problem of joint image segmentation and interpretation.

The main contribution of this paper is (i) the formulation of a general framework based on modular integration and multiresolution for solving computer vision tasks, and (ii) proposing a new joint image segmentation and interpretation algorithm to solve the problem of image interpretation. The layout of the paper is as follows: In $\S 2$, we give a formulation for a general vision task in the framework of modular integration and multiresolution and 
later use it for the formulation of the scene interpretation problem in $\S 2.2$. Section 3 details the scheme adopted to solve the scene interpretation problem, followed by a section (\$4) describing the experimental results to validate the proposed framework. We conclude in $\S 5$.

\section{Problem formulation}

\subsection{Generalized framework}

In the generalized framework, a vision task $\mathcal{V}$ can be considered to be performing the task of estimating an image attribute, say $\mathcal{A}$, from an observed image $\mathcal{Y}$ or a sequence of observed images. In many vision tasks, the observed image may not be used directly as it is, in estimating the attribute $\mathcal{A}$. In fact, in many vision tasks, it is some information derived from the observed image or images $\mathcal{Y}$ that is utilized. We refer to these variables derived from the observed image $\mathcal{Y}$ as core variables and denote them by $\mathcal{C}(\mathcal{Y})$. There is one more item, which often gets relegated to the list of assumptions used in solving a vision task, and this is the a priori knowledge. We denote the a priori domain knowledge by $\mathcal{K}$.

In order to present a computational formulation for the vision task $\mathcal{V}$, we need a relation between the attribute $\mathcal{A}$ and the pair $\{\mathcal{C}(\mathcal{Y}) ; \mathcal{K}\}$. In this paper, we assume a probabilistic relationship, namely, $\mathcal{P}[\mathcal{A}=a \mid \mathcal{C}(\mathcal{Y}) ; \mathcal{K}]$, the probability distribution of the attribute $\mathcal{A}$, given the core variables $\mathcal{C}(\mathcal{Y})$, and the domain knowledge $\mathcal{K}$ is either known or can be derived. We can now solve the attribute estimation problem as a maximum a posteriori (MAP) estimation problem. We can express the problem of solving the vision task $\mathcal{V}$ as:

Given (i) the core variables $\mathcal{C}(\mathcal{Y})$, (ii) a priori domain knowledge $\mathcal{K}$ and (iii) the relation $\mathcal{P}[\mathcal{A}=a \mid \mathcal{C}(\mathcal{Y}) ; \mathcal{K}]$, the optimal attribute $\mathcal{A}=a_{*}$ is obtained by solving the MAP estimation problem

$$
a_{*}=\arg \max _{a} \mathcal{P}[\mathcal{A}=a \mid \mathcal{C}(\mathcal{Y}) ; \mathcal{K}] .
$$

Most vision tasks are motivated by the way the human visual system (HVS) functions. Results obtained from experiments conducted on the visual systems of primates and experiments conducted in psychophysics and physiology of vision are used as factors to motivate the computational formulation of vision tasks.

Experiments from psychophysics and physiology of the visual system (Van Essen et al 1992) motivate us to look at the problem of solving the vision task $\mathcal{V}$ by breaking it into $m$ smaller tasks (called subtasks or modules) $\mathcal{V}_{1}, \mathcal{V}_{2}, \mathcal{V}_{3}, \ldots, \mathcal{V}_{m}$. Each subtask or module $\mathcal{V}_{i}$ interacts with other modules $\mathcal{V}_{j}$ - this is referred to as modular integration. Integration or synergism of modules is a technique where various modules get together to perform the given task better than when working individually with only feed-forward interaction. Synergistic integration of various modules has been used effectively in computer vision. Various paradigms have been used to integrate modules, for example Bozma \& Duncan (1994) use a game-theoretic approach to integrate vision modules, Clement \& Thonnat (1993) use a knowledge-based approach for integration, Gamble et al (1989) use MRF for labeling of surface discontinuities. Clark \& Yullie (1990) describe what they call strong 


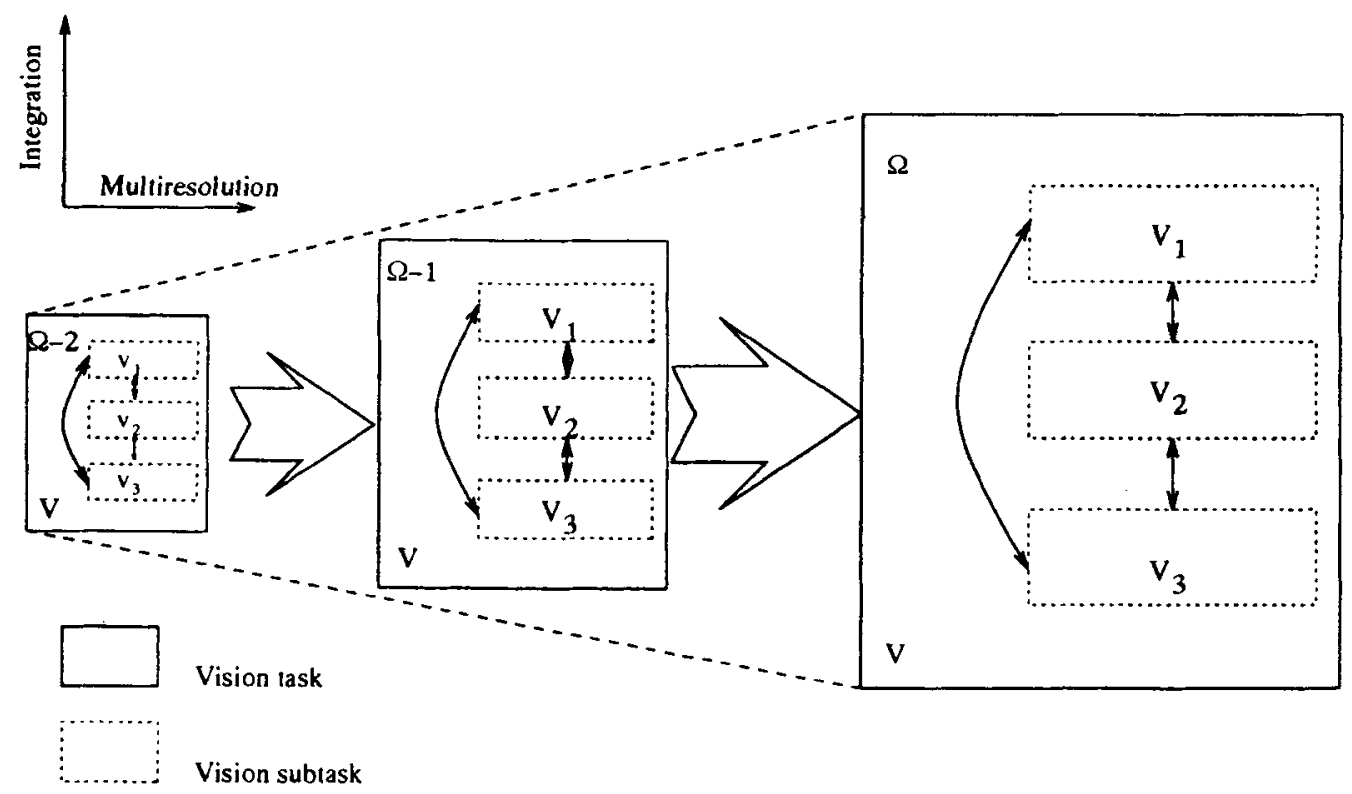

Figure 1. Modular integration is along the $y$-axis and the multiresolution in seen along the $x$-axis. The subtasks are shown as dotted rectangles and the vision task by solid rectangles. The largest solid rectangle is the vision task to be solved.

and weak integration of modules. Figure 1 shows the vision task $\mathcal{V}$ at any given resolution being divided into three subtasks $\mathcal{V}_{1}, \mathcal{V}_{2}$ and $\mathcal{V}_{3}$. The MAP estimation problem is solved for each module $\mathcal{V}_{i}, i=1,2,3$ to obtain the optimal attribute for each module. Depending on the vision task one or more of these attributes may be of interest.

Further, experimental results show that a multi-frequency channel decomposition seems to be taking place in the human visual cortex (Mallat 1989). Experiments based on adaptation techniques show that at some stage in the HVS, the visual information in different frequency bands is processed separately. It is also experimentally found that the retinal image seems to be decomposed into several frequency bands having approximately the same bandwidth on an octave scale. These experimental results motivate us to look at the vision task in a multiresolution framework. Multiresolution has been used extensively in literature to solve both image processing and computer vision problems (Rosenfeld 1984; Jolion \& Rosenfeld 1994).

Multiresolution is an efficient and effective way of representing data. The data at each resolution are the output of a bandpass filter with some centre frequency (usually the centre frequencies of the filters are one octave apart). The use of multiresolution is also motivated by the fact that the computational complexity of any vision task is large and multiresolution can be used effectively to reduce the computational complexity (Rosenfeld $1984 ; \mathrm{Li} \mathrm{1995).} \mathrm{Now,} \mathrm{the} \mathrm{problem} \mathrm{of} \mathrm{solving} \mathrm{the} \mathrm{vision} \mathrm{task} \mathcal{V}$ is reduced to the task of solving $\mathcal{V}$ at each resolution. Figure 1 depicts multiresolution approach along the $x$-axis. Let, $\mathcal{V}^{\Omega}$ represent the vision task at resolution $\Omega$. In the multiresolution approach the vision task $\mathcal{V}^{\Omega}$ is not solved directly at the resolution $\Omega$ but by solving a series of vision tasks $\mathcal{V}^{\Omega-n}, \mathcal{V}^{\Omega-n+1}, \ldots, \mathcal{V}^{\Omega-1}$ at coarser resolutions. 
Table 1. Some vision problems in the generalized framework.

\begin{tabular}{|c|c|c|c|c|}
\hline $\begin{array}{l}\text { Vision task } \\
\mathcal{V}\end{array}$ & $\begin{array}{l}\text { Modules } \\
\text { integrated }\end{array}$ & $\begin{array}{c}\text { Core variables } \\
\mathcal{C}(\mathcal{Y})\end{array}$ & $\begin{array}{c}\text { Knowledge } \\
\mathcal{K}\end{array}$ & $\begin{array}{l}\text { Attribute } \\
\qquad \mathcal{A}\end{array}$ \\
\hline $\begin{array}{l}\text { Colour image } \\
\text { restoration }\end{array}$ & $\begin{array}{l}\text { (i) Parameter } \\
\text { estimation, } \\
\text { (ii) Restoration }\end{array}$ & $\begin{array}{l}\text { Degraded colour } \\
\text { image }\end{array}$ & $\begin{array}{l}\text { (i) Degradation model } \\
y=A+\mathcal{W} \\
\text { (ii) } \mathcal{W} \text { is Gaussian }\end{array}$ & $\begin{array}{l}\text { Restored } \\
\text { image }\end{array}$ \\
\hline $\begin{array}{l}\text { Stereo } \\
\quad \text { vision }\end{array}$ & $\begin{array}{l}\text { (i) Feature } \\
\text { extraction } \\
\text { (ii) Matching } \\
\text { (iii) Interpolation }\end{array}$ & Stereo image pair & $\begin{array}{l}\text { (i) Precomputed edges } \\
\text { (ii) Disparity range } \\
\text { (iii) Disparity varies } \\
\text { smoothly } \\
\text { (iv) Epipolar constraint }\end{array}$ & $\begin{array}{l}\text { Disparity } \\
\text { or depth } \\
\text { map }\end{array}$ \\
\hline $\begin{array}{l}\text { Image } \\
\text { interpretation }\end{array}$ & $\begin{array}{l}\text { (i) Segmentation } \\
\text { (ii) Image } \\
\text { interpretation }\end{array}$ & $\begin{array}{l}\text { Grey levels, } \\
\text { scene based } \\
\text { features }\end{array}$ & $\begin{array}{l}\text { Nominal values of } \\
\text { gray level and shape } \\
\text { based features, and } \\
\text { spatial constraints }\end{array}$ & $\begin{array}{l}\text { Inter- } \\
\text { pretations }\end{array}$ \\
\hline
\end{tabular}

Motivated by the functioning of the HVS, we can express the problem of solving the vision task $\mathcal{V}^{\Omega}$ in the framework of modular integration and multiresolution as:

Given (i) the core variables $\mathcal{C}(\mathcal{Y})^{\Omega}$, (ii) a priori domain knowledge $\mathcal{K}^{\Omega}$ and (iii) the relation $\mathcal{P}\left[\mathcal{A}^{\Omega}=a^{\Omega} \mid \mathcal{C}(\mathcal{Y})^{\Omega} ; \mathcal{K}^{\Omega}\right]$. The optimal attribute $\mathcal{A}^{\Omega}=a_{*}^{\Omega}$ is obtained by solving the MAP estimation problem for $n=N, \ldots, 1$ (multiresolution) and $i=1, \ldots, m$ (modular integration)

$a_{i *}^{\Omega-n}=\arg \max _{a_{i}^{\Omega-n}} \mathcal{P}\left[\mathcal{A}_{i}^{\Omega-n}=a_{i}^{\Omega-n} \mid \mathcal{C}(\mathcal{Y})_{i}^{\Omega-n} ; \mathcal{K}_{i}^{\Omega-n}, \mathcal{A}_{j}^{\Omega-n}=\tilde{a}_{j}^{\Omega-n}, j \neq i\right]$

Of all the attributes $a_{i *}^{\Omega-n}, i=0,1, \ldots m$, one or more attributes ( $s a y a_{k *}^{\Omega-n}$ ) would correspond to the solution of the vision task $\mathcal{V}^{\Omega-n}$. The optimal solution $a_{k *}^{\Omega-n}$ obtained at resolution $\Omega-n$ is interpolated as:

$$
a_{k *}^{\Omega-n} \stackrel{\text { Interpolate }}{\longrightarrow} \tilde{a}_{k}^{\Omega-n+1}
$$

and is used to solve the vision task $\mathcal{V}^{\Omega-n+1}$ at the next finer resolution. $\tilde{a}_{k}^{\Omega-N}$ would correspond to some initial assignment of the attributes at the coarsest resolution $(\Omega-N)$.

Table 1 shows how the problems of colour image restoration, disparity estimation from stereo images and scene interpretation can fit into the proposed generalized framework. In this paper, we restrict ourselves to the problem of scene interpretation and solve this vision task using the developed framework. Details regarding colour image restoration in this generalized framework have been discussed by Nanda et al (1995) and those corresponding to disparity estimation using stereo images by Sunil Kumar \& Desai (1994).

Remark. Though not explicitly stated, the above problem formulation assumes that the conditional probability distribution $\mathcal{P}[\cdot \mid \cdot]$ is not only known at the finest resolution $\Omega$ but also retains its structure at coarser resolutions. An important issue in multiresolution 
computation of MRFs is how to preserve the Markovianity and define consistent model descriptions at different resolutions. In general, the local Markovian property is not preserved at the coarse levels after subsampling (Jeng 1992; Lakshmanan \& Derin 1993). For example if $\mathcal{P}^{\Omega}[\cdot \mid \cdot]$ is an MRF at resolution $\Omega$, then $\mathcal{P}^{\Omega-1}[\cdot \mid \cdot]$ need not be an MRF. Jeng (1992) gives the necessary and sufficient conditions for preserving the Markovianity and also states that there is at least one subsampling scheme by which Markovianity can be preserved ( $\mathrm{Li}$ 1995). A general transformation model is considered by Perez \& Heitz (1994) as the "restriction" of an MRF, defined on a finite arbitrary non-directed graph, to a subset of its original sites; several results are derived for the preservation of the Markovianity which may be useful for designing consistent and tractable multiresolution relaxation algorithms. Most often, we assume that $\mathcal{P}^{\Omega}[\cdot \mid \cdot]$ at the finest resolution $\Omega$, is an MRF. Moreover, at coarser resolution $(\Omega-n), \mathcal{P}^{\Omega}[\cdot \mid \cdot]$ can be approximated by an MRF. One such approximation is illustrated in the context of colour image restoration (Nanda et al 1995), where at each resolution, we learn the MRF model. Nevertheless, the general problem of MRF approximation at coarser resolution remains unresolved.

\subsection{Joint segmentation and image interpretation}

Scene understanding or image interpretation as seen in table 1 is a two-phase process consisting of a segmentation module and an interpretation module. These two modules are not entirely independent; good segmentation is a prerequisite for correct interpretation and on the other hand the knowledge of the scene (explicitly stated - the interpretation of the scene) is essential for good segmentation. This suggests that interaction should exist between the two modules. This fact was exploited first by Tenenbaum \& Barrow (1977) where they use interpretation to guide their segmentation algorithm. Sonka et al (1993) use a genetic algorithm to produce optimal image interpretations, they integrate segmentation and interpretation into a single feedback process that incorporates contextual knowledge. More recently, Kim \& Yang (1995) integrate segmentation and interpretation by forming a combined weighted energy function; the segmentation block is weighted high initially and then as the algorithm iterates the weights shift from the segmentation block to the interpretation block.

In this paper, initially we construct the wavelet transform of the image $\mathcal{Y}^{\Omega}$ (Mallat 1989) which results in $\mathcal{Y}^{\Omega-1}=D_{\mathcal{Y}, L L}^{\Omega-1}$, the low-pass filtered image and $D_{\mathcal{Y}, H L}^{\Omega-1}, D_{\mathcal{Y}, L H}^{\Omega-1}$, $D_{\mathcal{Y}, H H}^{\Omega-1}$ the difference image, each of size $2^{(\Omega-1)} \times 2^{(\Omega-1)}$. Figure 2 shows the wavelettransformed structure of $\mathcal{Y}^{\Omega}$, where $D_{\mathcal{Y}, H L}^{\Omega-1}\left(D_{\mathcal{Y}, L H}^{\Omega-1}\right)$ corresponds to the difference image obtained when $\mathcal{Y}^{\Omega}$ is filtered by a high-pass filter along the rows (columns) and by a lowpass filter along the columns (rows). The low-pass filtered image $\mathcal{Y}^{\Omega-1}$ is segmented using

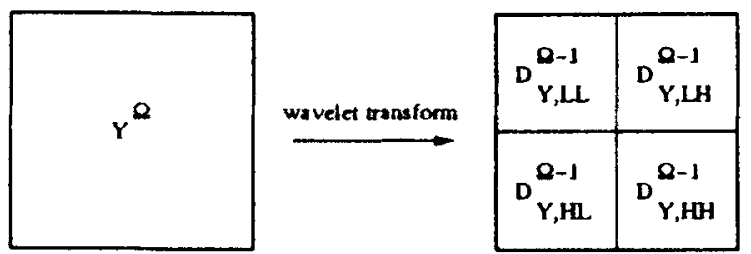

Figure 2. Wavelet transform representation of $Y^{\Omega}$. 


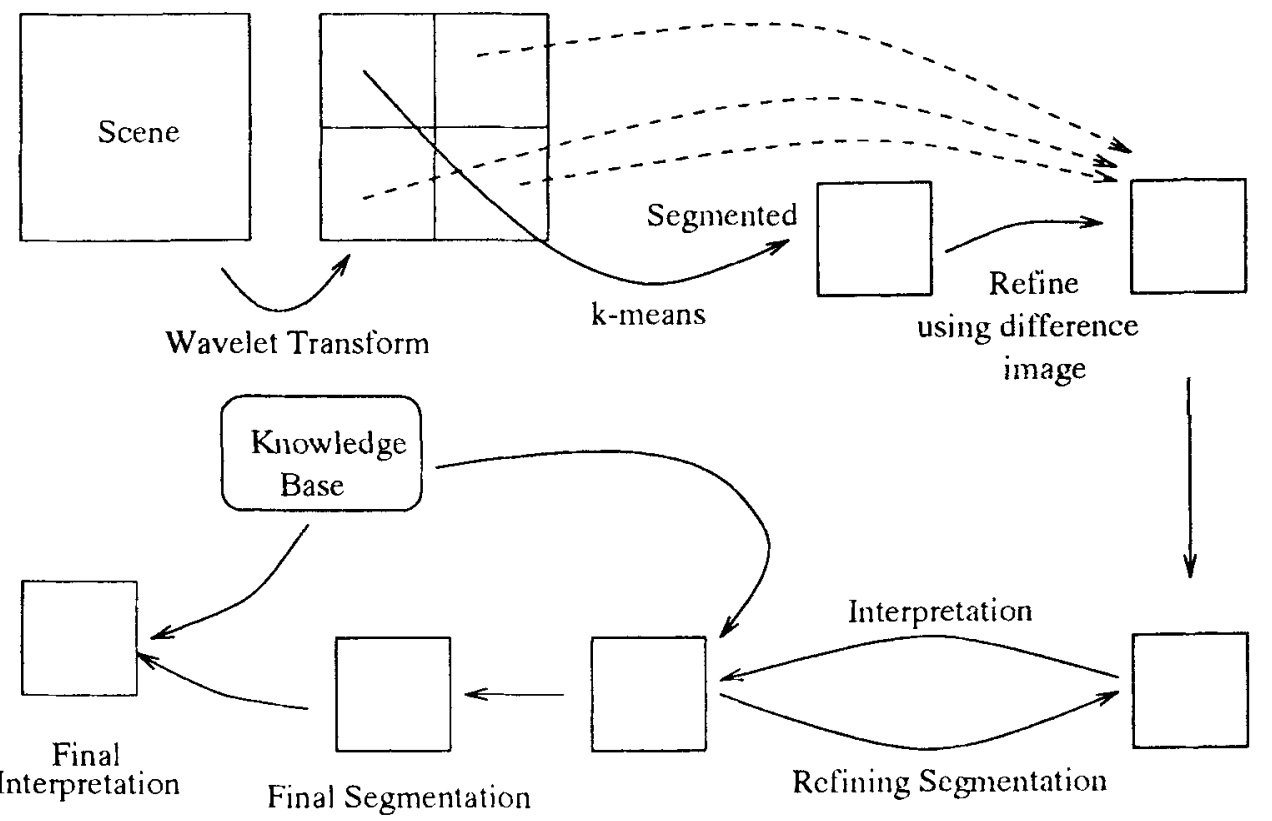

Figure 3. Macro-level joint segmentation and interpretation scheme.

any segmentation algorithm (in this paper for the purpose of simulations we have used the $k$-means clustering algorithm) to produce a crude segmented image. The segmented image is refined using the difference image $\left(D_{\mathcal{Y}, H L}^{\Omega-1}, D_{\mathcal{Y}, L H}^{\Omega-1}, D_{\mathcal{Y}, H H}^{\Omega-1}\right)$ described in $\S 3$.

The segmented image is subjected to interpretation. The problem of image interpretation is formulated in an MRF framework along the lines of Modestino \& Zhang (1992) except that we have a provision for a no-interpretation $\mathcal{L}_{0}$. The reason for having "nointerpretation" as a possible label is to refine the segmented image before further interpretation can be carried out. The process of interpretation, merging of the no-interpretation labels to produce a better segmented image and interpretation once again, is carried out until none of the regions have the label no-interpretation (see figure 3 ). The resulting segmented image is assumed to be the final segmented image and final interpretation is carried out on it.

At each resolution (say $k$ ) let the segmented image (see figure 4 ) be represented as an undirected simple planar graph. The nodes of the graph $\left(R_{1}^{k}, R_{2}^{k}, \ldots R_{n}^{k}\right)$ being represented by the $n$ regions in the segmented image and the edges of the graph represent the connectivity of the regions. Let $\mathcal{L}_{0}^{k}, \mathcal{L}_{1}^{k}, \mathcal{L}_{2}^{k}, \ldots, \mathcal{L}_{m}^{k}$ be the possible labels $\left(\mathcal{L}_{0}^{k} \rightarrow\right.$ no-interpretation label, and $\left\{\mathcal{L}_{i}^{k}\right\}_{i=1}^{m} \rightarrow$ the $m$ interpretation labels) and let the interpretation $\mathcal{I}_{j}^{k}$ be a random variable associated with the region $R_{j}^{k}, j=1, \ldots, n$, and $\mathcal{I}_{j}^{k}$ take a value from the label set $\left\{\mathcal{L}^{k}\right\} \triangleq\left\{\mathcal{L}_{0}^{k}, \mathcal{L}_{1}^{k}, \mathcal{L}_{2}^{k}, \ldots, \mathcal{L}_{m}^{k}\right\}$. Define $\mathcal{I}^{k}=\left[\mathcal{I}_{1}^{k}, \mathcal{I}_{2}^{k}, \ldots, \mathcal{I}_{n}^{k}\right]^{T}$. If $\mathcal{K}^{k}$ is the domain knowledge and $\mathcal{C}(\mathcal{Y})^{k}$ are the core variables obtained as a result of measurements made on the segmented image ${ }^{s} \mathcal{Y}^{k}$, then analogous to Modestino \& Zhang (1992) we assume that the conditional probability of $\mathcal{I}^{k}$, given $\mathcal{K}^{k}$ and $\mathcal{C}(\mathcal{Y})^{k}$ is a Markov Random Field, namely,

$$
\mathcal{P}\left[\mathcal{I}^{k} \mid \mathcal{K}^{k}, \mathcal{C}(\mathcal{Y})^{k}\right]=\left(1 / \mathcal{Z}^{k}\right) \exp \left[-U\left(\mathcal{I}^{k} ; \mathcal{K}^{k}, \mathcal{C}(\mathcal{Y})^{k}\right)\right]
$$


The Scene

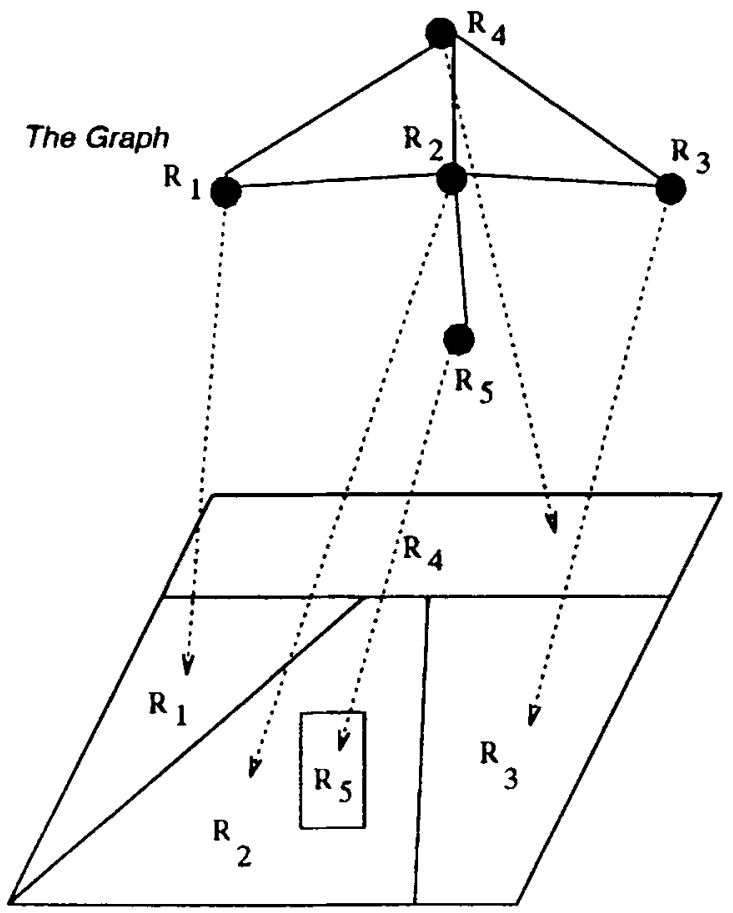

Figure 4. Segmented image represented as a graph.

is an MRF. The image interpretation problem is posed as an optimization problem and the optimal interpretation label vector $\mathcal{I}_{*}^{k}$ is obtained by solving the MAP estimation problem, namely,

$$
\begin{aligned}
\mathcal{I}_{*}^{k} & =\arg \max _{\mathcal{I}^{k} \in\left\{\mathcal{L}^{k}\right\}} \mathcal{P}\left[\mathcal{I}^{k} \mid \mathcal{K}^{k}, \mathcal{C}(\mathcal{Y})^{k}\right] \\
& =\arg \max _{\mathcal{I}^{k} \in\left\{\mathcal{L}^{k}\right\}} \frac{1}{Z^{k}} \exp \left[-U\left(\mathcal{I}^{k} ; \mathcal{K}^{k}, \mathcal{C}(\mathcal{Y})^{k}\right)\right] \\
& =\arg \min _{\mathcal{I}^{k} \in\left\{\mathcal{L}^{k}\right\}} U\left(\mathcal{I}^{k} ; \mathcal{K}^{k}, \mathcal{C}(\mathcal{Y})^{k}\right) .
\end{aligned}
$$

The problem of interpretation reduces to the problem of minimizing the energy function $U\left(\mathcal{I}^{k} ; \mathcal{K}^{k}, \mathcal{C}(\mathcal{Y})^{k}\right)$. The energy function is constructed such that it takes a minimum value when the interpretation labels are consistent with the knowledge $\mathcal{K}^{k}$ and the core variables $\mathcal{C}(\mathcal{Y})^{k}$ derived from ${ }^{s} \mathcal{Y}^{k}$. The minimization of the energy functional $U\left(\mathcal{I}^{k} ; \mathcal{K}^{k}, \mathcal{C}(\mathcal{Y})^{k}\right)$ results in interpretation of the given scene. Now,

$$
U\left(\mathcal{I}^{k} ; \mathcal{K}^{k}, \mathcal{C}(\mathcal{Y})^{k}\right)=\sum_{c \in \mathcal{C}} V_{c}\left(\mathcal{I}^{k} ; \mathcal{K}^{k}, \mathcal{C}(\mathcal{Y})^{k}\right)
$$

where $V_{c}(\cdot ; \cdot, \cdot)$ 's are the clique functions (Geman \& Geman 1984) which need to be constructed. The clique function should decrease when the interpretation labels are consistent with the domain knowledge and core variables, thus resulting in a decrease of the energy function. This means that the interpretation of the image is the one most consistent with the domain knowledge and core variables will have minimum energy. For completeness, 


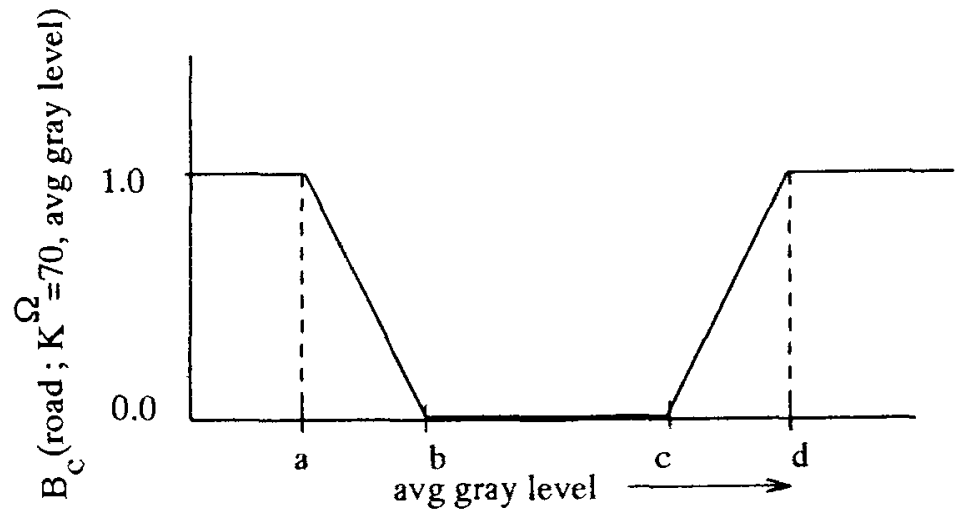

Figure 5. Example of a basis function used for the average gray level feature for road (also see table 4 in $\$ 4.2$ ). Here, $a=60, b=65, c=75, d=80$.

we present the clique functions for 1-node and 2-node cliques (Modestino \& Zhang 1992).

\subsection{Construction of clique functions}

Single or 1-node clique functions: Let $c$ be an arbitrary single node clique corresponding to the region $\eta$, with a single node $R_{\eta}^{k}$. Let $V_{c}\left(\mathcal{I}_{\eta}^{k} ; \mathcal{K}^{k}, \mathcal{C}(\mathcal{Y})^{k}{ }_{\eta}\right)$ be the corresponding clique function. Let there be $p$ features associated with core variables $\mathcal{C}(\mathcal{Y})_{\eta}^{k}=\left[{ }^{1} \mathcal{C}(\mathcal{Y})_{\eta}^{k},{ }^{2} \mathcal{C}(\mathcal{Y})_{\eta}^{k}\right.$, $\left.\ldots,{ }^{p} \mathcal{C}(\mathcal{Y})_{\eta}^{k}\right]$. Assume these core variables to be independent ${ }^{2}$, then the clique function can be defined as

$$
V_{c}\left(\mathcal{I}_{\eta}^{k} ; \mathcal{K}^{k}, \mathcal{C}(\mathcal{Y})_{\eta}^{k}\right)=\sum_{i=1}^{p} W_{c}^{i} B_{c}^{i}\left(\mathcal{I}_{\eta}^{k} ; \mathcal{K}^{k},{ }^{i} \mathcal{C}(\mathcal{Y})_{\eta}^{k}\right)
$$

where $W_{c}^{i}$ are positive constants called weights, such that $\sum_{i=1}^{p} W_{c}^{i}=1$ and $B_{c}^{i}(\cdot ; \cdot, \cdot)$ is the basis function associated with the feature $i$, which satisfies the requirement of the clique function, namely, that it should take a small value when the interpretation labels are consistent with the domain knowledge and the core variables. Now, the construction of the clique function reduces to the construction of a basis function. The choice of the basis function could come from $\mathcal{P}\left[\mathcal{I}^{k} \mid \mathcal{K}^{k}, \mathcal{C}(\mathcal{Y})^{k}\right]$ suitably modified to adhere to the requirement mentioned above ${ }^{3}$, or we could use a piecewise linear basis function (Modestino \& Zhang 1992). An example is illustrated in figure 5 for the average gray level feature for road.

2-node clique functions: The construction of a multiple node clique function is similar in philosophy to the 1-node clique except that it has two types of basis functions. The first one is similar to that of the 1-node clique function, and depends on the feature measurements (core variables); the second part depends on the spatial constraints (like a car can be in the neighbourhood of a road but not in the neighbourhood of the sky).

\footnotetext{
${ }^{2}$ If they are not independent, we could "merge" core variables which are dependent into one core variable and work with a core variable set of smaller size

${ }^{3}$ Since $P\left[\mathcal{I}^{k} \mid \mathcal{K}^{k}, \mathcal{C}(\mathcal{Y})^{k}\right]$ takes a value near 1 when the interpretation labels coincide with $\mathcal{K}^{k}, \mathcal{C}(\mathcal{Y})^{k}$, we have to properly modify it. We could use, for example, $-\alpha_{c} \log P\left[\mathcal{I}^{k} \mid \mathcal{K}^{k}, \mathcal{C}(\mathcal{Y})^{k}\right]$
} 
Table 2. Rules for constructing knowledge pyramid.

\begin{tabular}{lcc}
\hline Features & $\mathcal{K}^{\Omega-\xi}$ & $\mathcal{K}^{\Omega-\xi-1}$ \\
\hline Area & $A$ & $A / 4$ \\
Perimeter & $\mathrm{P}$ & $\mathrm{P} / 2$ \\
Average gray value & $\mathrm{G}$ & $\mathrm{G}$ \\
Mass center & $\mathrm{M}$ & $M / 2$ \\
Variance & $\mathrm{V}$ & $\mathrm{V}$ \\
Compactness & $\mathrm{C}$ & $\mathrm{C}$ \\
Contrast & $\mathrm{CR}$ & $\mathrm{CR}$ \\
Common perimeter ratio & $\mathrm{CPR}$ & $\mathrm{CPR}$ \\
\hline & & \\
$V_{c}\left(\mathcal{I}_{c}^{k} ; \mathcal{K}^{k}, \mathcal{C}(\mathcal{Y})^{k}\right)=\sum_{i=1}^{q} \bar{W}_{c}^{i} \bar{B}_{c}^{i}\left(\mathcal{I}_{c}^{k} ; \mathcal{K}^{k},{ }^{i} \mathcal{C}(\mathcal{Y})_{k}^{k}\right)+S_{c}\left(\mathcal{I}_{c}^{k}, \mathcal{C}(\mathcal{Y})_{c}^{k}\right)$,
\end{tabular}

where, $\sum_{i}^{q} \bar{W}_{c}^{i}=1$ and $\left.S_{c}\left(\mathcal{I}_{c}^{k}, \mathcal{C}(\mathcal{Y})_{c}^{k}\right)\right) \triangleq \alpha_{c}\left(1-\mathcal{P}\left[\mathcal{I}_{c}^{k} \mid \mathcal{K}^{k}\right]\right)$, such that,

$$
\mathcal{P}\left[\mathcal{I}_{c}^{k} \mid \mathcal{K}^{k}\right]= \begin{cases}1, & \text { if } \mathcal{I}_{c}^{k} \text { is a valid combination according to } \mathcal{K}^{k}, \\ 0, & \text { if } \mathcal{I}_{c}^{k} \text { is not a valid combination according to } \mathcal{K}^{k}\end{cases}
$$

Note, $c$ is a 2 -node clique and $\left.S_{c}\left(\mathcal{I}_{c}^{k}, \mathcal{C}(\mathcal{Y})_{c}^{k}\right)\right)=0$ represents a valid combination, while $\left.S_{c}\left(\mathcal{I}_{c}^{k}, \mathcal{C}(\mathcal{Y})_{c}^{k}\right)\right)=1$ represents an invalid combination.

\section{The joint segmentation and image interpretation scheme}

The proposed scheme of joint segmentation and image interpretation is pictorially depicted for two resolutions in figure 6 . The description of the algorithm is based on figure 6 .

Step 0: Initialization: Given, $\mathcal{K}^{\Omega}$ the a priori knowledge and $\mathcal{Y}^{\Omega}$ (figure 6 , component a), the scene to be interpreted, defined on a lattice of size $2^{\Omega} \times 2^{\Omega}$, construct $D_{\mathcal{Y}, L L}^{\Omega-1}, D_{\mathcal{Y}, H H}^{\Omega-1}$, $D_{\mathcal{Y}, H L}^{\Omega-1}, D_{\mathcal{Y}, L H}^{\Omega-1}, D_{\mathcal{Y}, H H}^{\Omega-2}, D_{\mathcal{Y}, H L}^{\Omega-2}, D_{\mathcal{Y}, L H}^{\Omega-2}$ using a wavelet filter (figure 6, component b).

Step I: Segmentation and refining: Obtain ${ }^{s} \tilde{\mathcal{Y}}^{\Omega-\xi}$ the segmented image of $\mathcal{Y}^{\Omega-\xi} \triangleq D_{\mathcal{Y}, L L}^{\Omega-\xi}$ using $k$-means clustering algorithm (figure 6 , component c) and refine ${ }^{s} \tilde{\mathcal{Y}}^{\Omega-\xi}$, (i) using $D_{\mathcal{Y}, H H}^{\Omega-\xi}, D_{\mathcal{Y}, H L}^{\Omega-\xi}$ and $D_{\mathcal{Y}, L H}^{\Omega-\xi}$, and (ii) using a predefined threshold to merge all segments whose area is less than the prespecified minimum area, to get ${ }^{s} \mathcal{Y}^{\Omega-\xi}$ (figure 6, component d).

(1) At the coarsest resolution $\Omega-N$ : (a) Construct a histogram of $\mathcal{Y}^{\Omega-N}$, choose dominant peaks - let there be $B$ such peaks. These are the chosen values of the bins in the $k$-means clustering algorithm.

(b) $k$-means clustering algorithm will produce, say, some $M$ segments using the optimality criterion, namely, that the pixels which are within a region are as close as possible to the centroid of the region.

(c) Refine the regions or segments obtained from Step (I.1.b) using the difference images $D_{\mathcal{Y}, H H}^{\Omega-N}, D_{\mathcal{Y}, H L}^{\Omega-N}$ and $D_{\mathcal{Y}, L H}^{\Omega-N}$ and the minimum area criteria. 


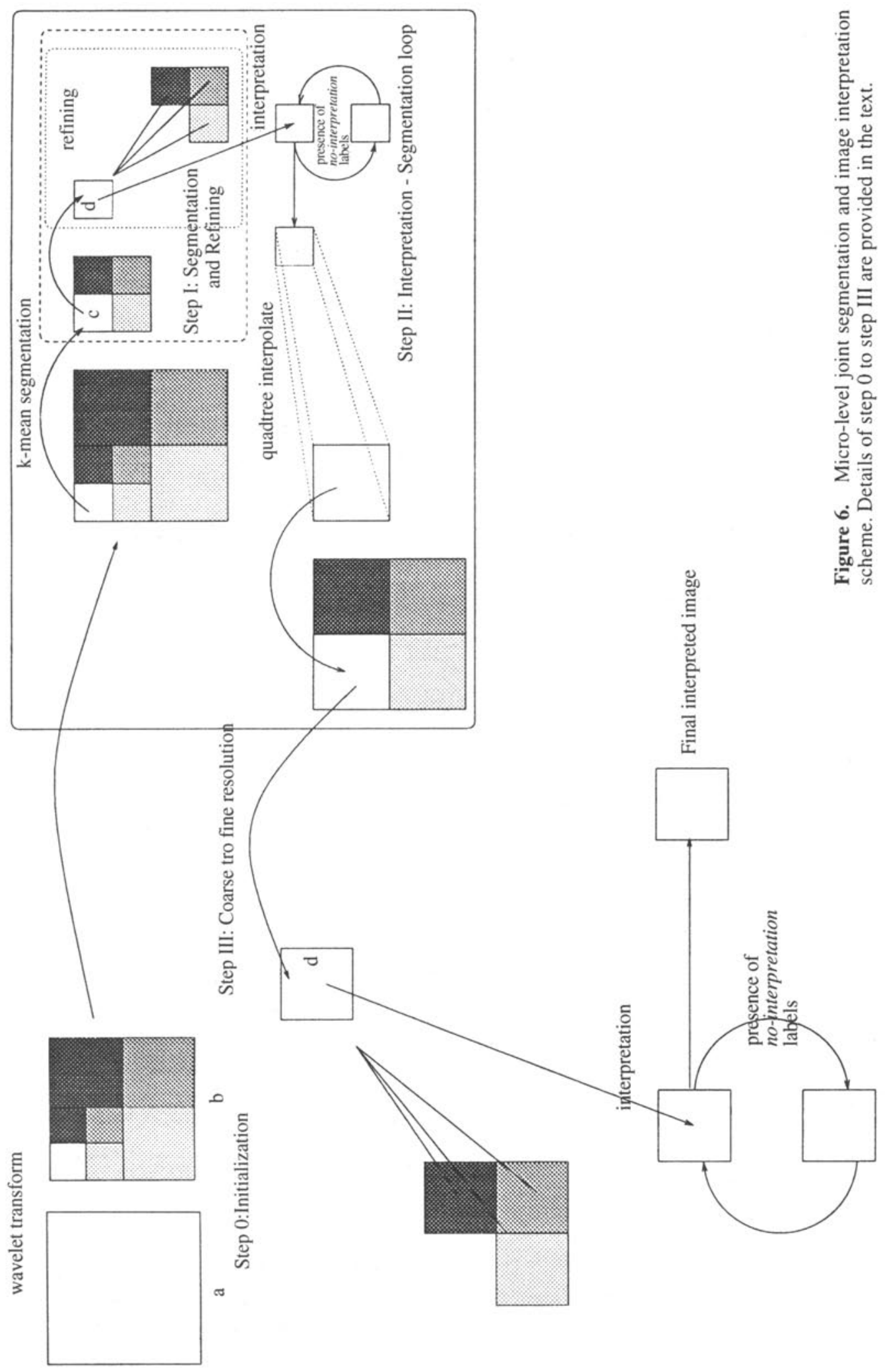




\begin{tabular}{|c|c|c|}
\hline$i-1, j-1$ & $i-1, j$ & $i-1, j+1$ \\
\hline$i, j-1$ & $i, j$ & $i, j+1$ \\
\hline$i+1, j-1$ & $i+1, j$ & $i+1, j+1$ \\
\hline
\end{tabular}

Figure 7. To describe the process of refining segmentation.

(i) Refinement using the difference image: The refinement procedure is best described by looking at figure 7 . For example if $D_{\mathcal{Y}, H H}^{\Omega-N}$ is not zero at the pixel location $(i, j)$ it means that there is a diagonal edge present at the pixel location $(i, j)$. The presence of an edge means that pixels $(i-1, j-1)$ and $(i, j)$ should not belong to the same segment. If the pixels $(i-1, j-1)$ and $(i, j)$ belong to different clusters they are not touched, else pixel $(i-1, j-1)$ is assigned a new segment which is not the same as that occupied by pixel $(i, j)$. The assignment of the segment label is based on the nearest neighborhood scheme: pixel $(i-1, j-1)$ is assigned to that segment whose centroid is closest to the gray level value of the pixel $(i-1, j-1)$ and excluding the centroid of the segment to which pixel $(i, j)$ belongs. In a similar manner, refinement is done using the difference images $D_{\mathcal{Y}, L H}^{\mathcal{S}-N}$ and $D_{\mathcal{Y}, H L}^{\Omega-N}$ corresponding to the vertical and horizontal edge fields respectively.

(ii) Refinement using the minimum area criterion: Let $k$ be a region with an area less than the minimum area and let $l, m$ be regions adjacent to region $k$ and having areas greater than the minimum area. Then the refinement criterion is:

region $k$ is merged with region $l$ if the difference between the gray level of the centroids of regions $k$ and $l$ is less than the difference between the gray level of the centroids of regions $k$ and $m$.

In other words, if $R_{q}$ represents region $q$ and $G\left(R_{q}\right)$ represents the average gray level value of region $R_{q}$, then the above criterion translates as:

$$
\begin{array}{lll}
R_{i} \longleftarrow R_{k} \uplus R_{i} & \text { if } & \left|G\left(R_{k}\right)-G\left(R_{i}\right)\right| \leq\left|G\left(R_{k}\right)-G\left(R_{j}\right)\right|, \\
R_{j} \longleftarrow R_{k} \uplus R_{j} & \text { if } \quad\left|G\left(R_{k}\right)-G\left(R_{i}\right)\right| \geq\left|G\left(R_{k}\right)-G\left(R_{j}\right)\right|,
\end{array}
$$

where, the notation $R_{i} \longleftarrow R_{l} \uplus R_{i}$ means that region $R_{i}$ and $R_{l}$ are merged to form a new region $R_{i}$.

(2) At any resolution $\Omega-\xi(\xi \neq N)$ : (a) Quadtree interpolate the segmented image at $(\Omega-\xi-1)$ to $(\Omega-\xi)$ resolution. If ${ }^{s} \mathcal{Y}_{i j}^{\Omega-\xi-1}$ is the $(i, j)$ th pixel of the segmented image at resolution $(\Omega-\xi-1)$, then

$$
{ }^{s} \mathcal{Y}_{i+p, j+q}^{\Omega-\xi}={ }^{s} \mathcal{Y}_{i, j}^{\Omega-\xi-1} \text { for } 0 \leq p, q \leq 1 .
$$

Use this information as the initial segmented image. In addition the centroids of each of the regions is also transferred to the next finer resolution. These centroids are then used to initialize the bins to be used in the k-means clustering algorithm at resolution $(\Omega-\xi)$. 
(b) Use the interpretation labels obtained from the previous resolution $\Omega-\xi-1$, to initialize the interpretation labels at this resolution.

(c) Repeat (I.1.b) and (I.1.c) for resolution $(\Omega-\xi)$.

Step II: Interpretation - segmentation loop: (1) Interpretation - The segments are interpreted using the knowledge base $\mathcal{K}^{\Omega-\xi}$, the core variables $\mathcal{C}(\mathcal{Y})^{\Omega-\xi}$ derived from the segmented image ${ }^{s} \mathcal{Y}^{\Omega-\xi}$, and the assumption that the conditional probability is an MRF, (1). This conditional probability can be minimized using a relaxation algorithm (in our simulations we used the simulated annealing algorithm). The energy to be minimized is

$$
\arg \min _{\mathcal{I}^{\Omega-\xi} \in\left\{\mathcal{L}^{\Omega-\xi}\right\}} U\left(\mathcal{I}^{\Omega-\xi} ; \mathcal{K}^{\Omega-\xi}, \mathcal{C}(\mathcal{Y})^{\Omega-\xi}\right),
$$

where,

$$
U\left(\mathcal{I}^{\Omega-\xi} ; \mathcal{K}^{\Omega-\xi}, \mathcal{C}(\mathcal{Y})^{\Omega-\xi}\right)=\sum_{c \in \mathcal{C}} V_{c}\left(\mathcal{I}^{\Omega-\xi} ; \mathcal{K}^{\Omega-\xi}, \mathcal{C}(\mathcal{Y})^{\Omega-\xi}\right),
$$

and $V_{c}\left(\mathcal{I}^{\Omega-\xi} ; \mathcal{K}^{\Omega-\xi}, \mathcal{C}(\mathcal{Y})^{\Omega-\xi}\right)$ are the clique potentials (see $\S 2.3$ ).

(2) Recall - that $\left\{\mathcal{L}^{\Omega-\xi}\right\}$ contains the no-interpretation label. Thus our criterion for moving from coarse to fine resolution is:

if none of the labels have the label no-interpretation then move from the present coarse resolution to the next fine resolution (step III).

(3) Segmentation - In case any segment has the label no-interpretation, we merge it with one of the interpreted segments which is adjacent to it, depending on a probability criterion. For example: If region $j$ has label no-interpretation, that is, $\mathcal{I}_{j}^{\Omega-\xi}$ takes the label $\mathcal{L}_{0}$ and if $l, m$ are the regions adjacent to region $j$, then region $j$ is assigned the interpretation label $\mathcal{L}_{l}$ corresponding to the region $l$ if $\mathcal{P}\left[\mathcal{I}_{j}^{\Omega-\xi}=\mathcal{L}_{l} \mid \mathcal{C}(\mathcal{Y})^{\Omega-\xi} ; \mathcal{K}^{\Omega-\xi}\right]>\mathcal{P}\left[\mathcal{I}_{j}^{\Omega-\xi}=\mathcal{L}_{m} \mid\right.$ $\left.\mathcal{C}(\mathcal{Y})^{\Omega-\xi} ; \mathcal{K}^{\Omega-\xi}\right]$. In other words,

$$
\mathcal{I}_{j}^{\Omega-\xi}=\left\{\begin{array}{rrrr}
\mathcal{L}_{l} \quad \text { if } & \mathcal{P}\left[\mathcal{I}_{j}^{\Omega-\xi}=\mathcal{L}_{l} \mid \mathcal{K}^{\Omega-\xi} ; \mathcal{C}(\mathcal{Y})^{\Omega-\xi}\right]> \\
& & & \mathcal{P}\left[\mathcal{I}_{j}^{\Omega-\xi}=\mathcal{L}_{m} \mid \mathcal{K}^{\Omega-\xi} ; \mathcal{C}(\mathcal{Y})^{\Omega-\xi}\right] \\
\mathcal{L}_{m} \quad \text { if } & \mathcal{P}\left[\mathcal{I}_{j}^{\Omega-\xi}=\mathcal{L}_{m} \mid \mathcal{K}^{\Omega-\xi} ; \mathcal{C}(\mathcal{Y})^{\Omega-\xi}\right]> \\
& & & \mathcal{P}\left[\mathcal{I}_{j}^{\Omega-\xi}=\mathcal{L}_{l} \mid \mathcal{K}^{\Omega-\xi} ; \mathcal{C}(\mathcal{Y})^{\Omega-\xi}\right]
\end{array}\right.
$$

This step will output a better segmented image.

(4) Go back to step II (interpretation-segmentation loop).

Step III: Coarse to fine resolution: (1) If not working at $\Omega-1$ resolution: (a) Interpretation labels at this resolution are transferred to the next finer resolution $\left(\mathcal{I}^{\Omega-\xi} \longrightarrow \mathcal{I}^{\Omega-\xi+1}\right)$, (b) $\xi \longrightarrow \xi-1$; go back to Step I (segmentation and refining).

(2) At resolution $\Omega-1$ : (a) Output the interpretation labels,

(b) output the segmented image.

(c) Quadtree interpolate the segmented and interpreted images to obtain the final segmented and interpreted images at the finest resolution $\Omega$ (in all our experiments, we have found that it was sufficient to stop the algorithm at resolution $\Omega-1$ instead of segmenting and interpreting the image at resolution $\Omega$ ). 
Table 3. Definitions of features used in our simulations. (figure 8 shows these features with an example).

\begin{tabular}{ll}
\hline Feature & \multicolumn{1}{c}{ Definition } \\
\hline Area $(A)$ & Total number of pixels in the region $R$ \\
Perimeter $(P)$ & Total number of pixels on the boundary of segment \\
Average gray value $(G)$ & $\frac{1}{A} \sum_{i, j \in R} x(i, j)$, where $x(i, j)$ are the \\
gray levels of the pixel at the location $(i, j)$ & \\
Mass centre $\left(m_{x}, m_{y}\right)$ & $\mathrm{M}=\left\{m_{x}, m_{y}\right\}=\left\{\frac{1}{A} \sum_{i, j \in R} i, \frac{1}{A} \sum_{i, j \in R} j\right\}$ \\
Variance $(V)$ & $\left\{\frac{1}{A} \sum_{i, j \in R}(x(i, j)-G)^{2}\right\}^{1 / 2}$ \\
Compactness $(C)$ & $P^{2} / 4 \pi A$ \\
Contrast $(C R)$ & $\left|G_{i}-G_{j}\right|$, regions $i, j$ are adjacent, \\
& and $G_{i}$ represents the average gray value of region $i$ \\
Common perimeter ratio $(C P R)$ & $\frac{P_{i j}}{P_{i}+P_{j}}$, where $P_{i j}$ is the common perimeter $(C P)$ \\
& to region $i$ and $j$ \\
\hline
\end{tabular}

Remark 1. Quadtree interpolation is simple and fast compared to the usual inverse wavelet transformation. Also, wavelet interpolation did not provide any appreciable advantage.

Remark 2. In principle it may be possible to generate the knowledge base separately at each resolution. But, knowledge base generation is a user interactive process, which tends to get difficult at coarser resolutions. Thus, we prefer to use the rules described in table 2 for constructing the knowledge pyramid (figure 8 shows the features with an example).

\section{Experimental results}

Experiments were carried out to validate the proposed scheme of joint segmentation and image interpretation in the framework of modular integration and multiresolution. Tests were conducted both on real outdoor (road images) and indoor (computer images) images 4 of size $256 \times 256$ which were either captured using the QuickTake100 digital camera (figures 9 and 10) or using an aim and shoot Kodak Pro 111 camera and then scanning using the HP Color Scanner (figures 9-11).

\subsection{Features used}

Feature selection is an important aspect of image interpretation. The features that are used can be broadly classified as (i) primary features, namely the features that are obtained from the scene through direct measurement and (ii) secondary features are the features that are derived from primary features and hence are not directly measured from the scene.

\footnotetext{
$\overline{{ }^{4} \text { url http://144.16.100.30/images/Interpretation/ }}$
} 


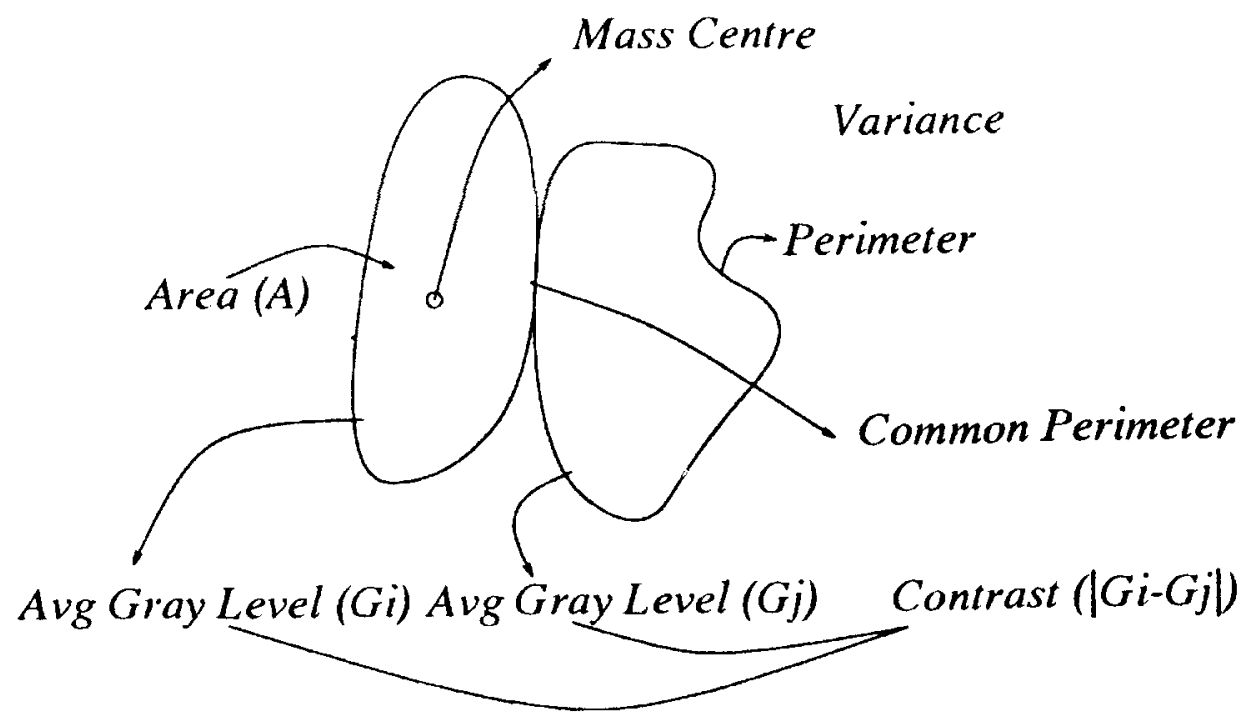

Figure 8. Features used in scene interpretation (table 3 gives their mathematical definition).

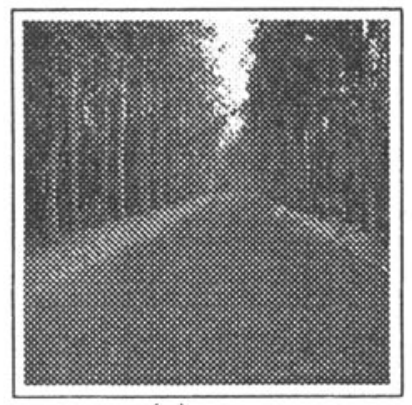

(a)

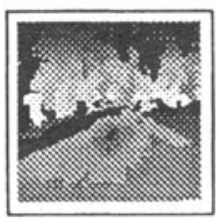

(c)

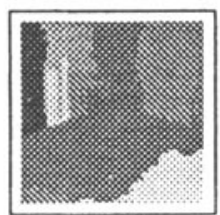

(d)

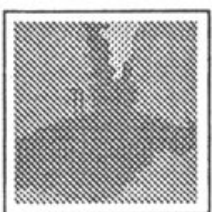

(e)

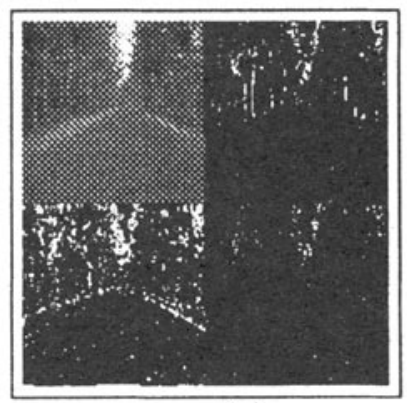

(b)

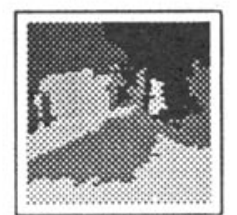

(f)

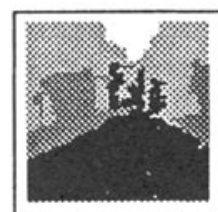

(g)

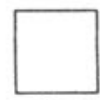

sky
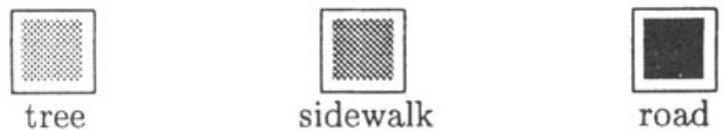

road

Figure 9. (a) Original scene image of size $256 \times 256$; (b) wavelet transforned image; (c) initial segmentation (after $k$-means clustering) of size $128 \times 128$; (d) segmentation at an intermediate stage; (e) interpretation at an intermediate stage; (f) final segmentation of size $128 \times 128$; $(\mathrm{g})$ final interpretation, and the interpretation legends corresponding to $(\mathrm{g})$. 
Table 4. Knowledge base used for the road images (figures 9 and 10).

\begin{tabular}{|c|c|c|c|c|}
\hline Label & $\mathrm{A}$ & G & $\mathbf{P}$ & $m_{x}$ \\
\hline$\overline{\text { Sky }}$ & 1052 & 180 & 204 & 63 \\
\hline Tree & 3087 & 64 & 312 & 18 \\
\hline Sidewalk & 1858 & 99 & 496 & 54 \\
\hline Road & 6193 & 70 & 411 & 66 \\
\hline Common perimeter $(C P)$ & Sky & Tree & Sidewalk & Road \\
\hline Sky & - & 93 & - & - \\
\hline Tree & 93 & - & 479 & - \\
\hline Sidewalk & - & 479 & - & 508 \\
\hline$\underline{\text { Road }}$ & - & - & 508 & - \\
\hline $\begin{array}{l}\mathcal{I}, \mathcal{C}(\mathcal{Y}) \rightarrow \\
\downarrow\left\{S_{c}(\mathcal{I}, \mathcal{C}(\mathcal{Y}))\right\} \\
\end{array}$ & Sky & Tree & Sidewalk & Road \\
\hline Sky & 1 & 0 & 1 & 1 \\
\hline Tree & 0 & 1 & 0 & 1 \\
\hline Sidewalk & 1 & 0 & 1 & 0 \\
\hline Road & 1 & 1 & 0 & 1 \\
\hline
\end{tabular}

The features used in all our experimental work are (table 3): (i) Single node cliques: area $(A)$, perimeter $(P)$, average grey level $(G)$, the mass centre $\left(m_{x}, m_{y}\right)$, variance $(V)$, compactness $(C)$, and (ii) Two node cliques: contrast $(C R)$, common perimeter ratio or boundary length $(C P R)$.

\subsection{Road images}

Figures $9 \mathrm{a}$ and $10 \mathrm{a}$ are the original images of the scene to be interpreted and figures $9 \mathrm{~b}$ and $10 \mathrm{~b}$ are the wavelet-transformed images of figures $9 \mathrm{a}$ and $10 \mathrm{a}$ respectively using the 4-tap Daubechies filter coefficients. The $D_{\mathcal{Y}, L L}^{\Omega-1}$ is segmented using the $k$-means clustering algorithm and refined using $D_{\mathcal{Y}, H L}^{\Omega-1}, D_{\mathcal{Y}, L H}^{\Omega-1}$ and $D_{\mathcal{Y}, H H}^{\Omega-1}$ as described in $\S 3$ (see figure 2). The resulting image is displayed in figures $9 \mathrm{c}$ and $10 \mathrm{c}$. Figures $9 \mathrm{~d}$ and $9 \mathrm{e}$ depict segmentation and interpretation of the scene at an intermediate stage. The final segmented image is shown in figures $9 \mathrm{f}$ and $10 \mathrm{f}$. The final interpreted image is shown in figures $9 \mathrm{~g}$ and $10 \mathrm{~g}$. The a priori knowledge (details regarding the acquisition of knowledge are given by Sunil Kumar \& Desai 1996) that is used for interpreting the images in this section is tabulated in table 4.

In figure $9 \mathrm{f}$ the segmentation is not perfect, for example the road is in fact divided into 2 different segments, but the interpretation block interprets both the segments as road and this is seen in figure $9 \mathrm{~g}$. This is an indication that the segmentation and the interpretation modules cannot work independently, they work best when they work synergetically. This aspect of modular integration is also seen in figure $10 \mathrm{~g}$, where the segments corresponding to sky (see figure 10f) are all merged into a single segment after interpretation. 


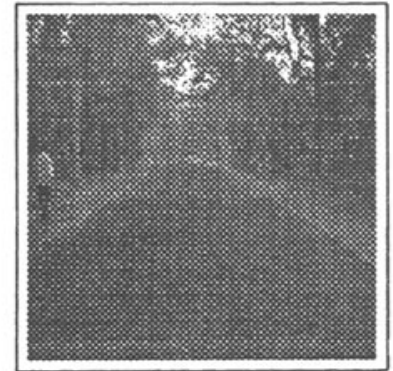

(a)

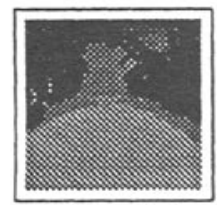

(c)

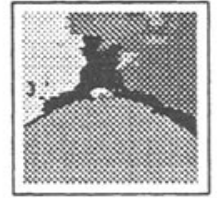

(d)

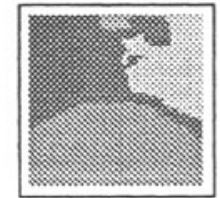

(e)

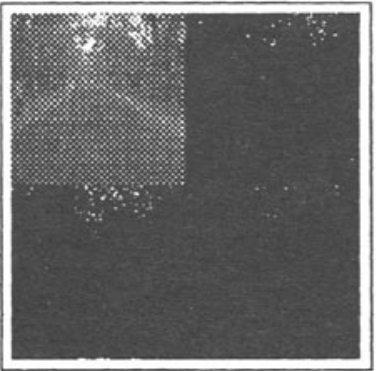

(b)

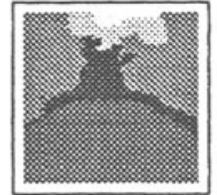

(f)

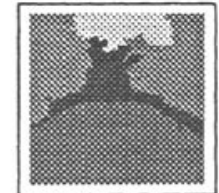

(g)
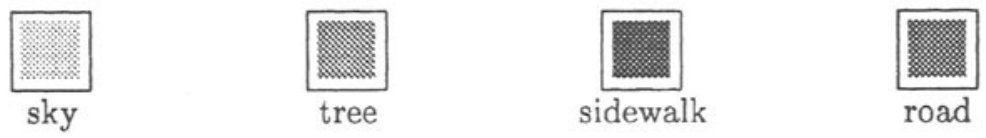

Figure 10. (a) Original scene image of size $256 \times 256$ : (b) wavelet transformed image: (c) initial segmentation (after $k$-means clustering) of size $128 \times 128$; (d) segmentation at an intermediate stage: (e) interpretation at an intermediate stage: (f) final segmentation of size $128 \times 128:(\mathrm{g})$ final interpretation, and the interpretation legends corresponding to $(\mathrm{g})$.

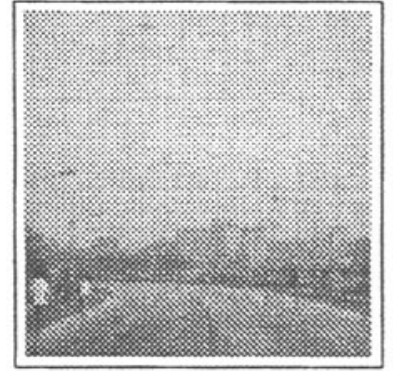

(a)

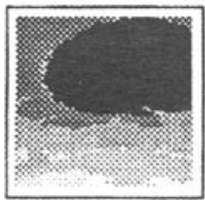

(c)

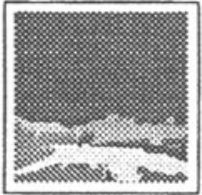

(d)

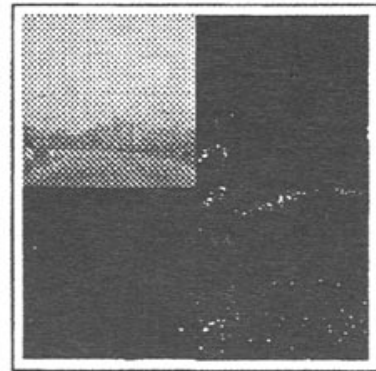

(b)

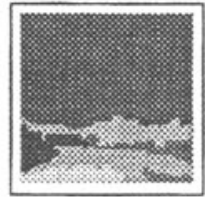

(e)

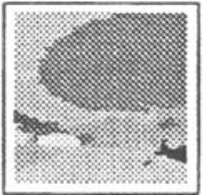

(f)

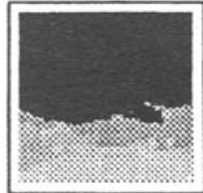

(g)
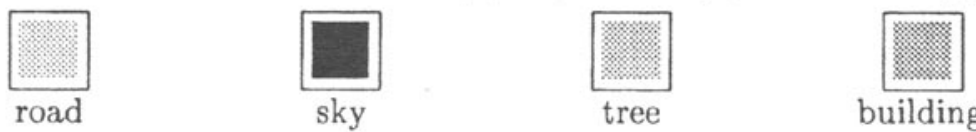

building

Figure 11. (a) Original scene image of size $256 \times 256$ : (b) wavelet transformed image; (c) initial segmentation (after $k$-means clustering) of size $128 \times 128$; (d) segmentation at an intermediate stage; (e) interpretation at an intermediate stage; (f) final segmentation of size $128 \times 128 ;(\mathrm{g})$ final interpretation, and the interpretation legends corresponding to $(\mathrm{g})$. 
Table 5. Knowledge base used for outdoor building (build) scene (figure 11).

\begin{tabular}{|c|c|c|c|c|c|c|}
\hline Label & A & $\mathrm{G}$ & $P$ & $m_{x}$ & $m_{y}$ & $\mathrm{~V}$ \\
\hline Road & 4999 & 106 & 395 & 64 & 107 & 18.334378 \\
\hline Sky & 7730 & 208 & 443 & 64 & 30 & 20.975156 \\
\hline Tree (Left) & 1535 & 85 & 285 & 37 & 80 & 17.957333 \\
\hline Tree (Right) & 550 & 78 & 113 & 117 & 84 & 14.810193 \\
\hline Build (Left) & 879 & 137 & 204 & 24 & 63 & 19.207092 \\
\hline Build (Right) & 685 & 145 & 127 & 108 & 65 & 15.920010 \\
\hline $\begin{array}{l}\text { Common } \\
\text { perimeter }(C P)\end{array}$ & Road & Sky & $\begin{array}{l}\text { Tree } \\
\text { (Left) }\end{array}$ & $\begin{array}{c}\text { Tree } \\
\text { (Right) }\end{array}$ & $\begin{array}{l}\text { Build } \\
\text { (Left) }\end{array}$ & $\begin{array}{l}\text { Build } \\
\text { (Right) }\end{array}$ \\
\hline Road & - & - & 192 & 89 & - & 29 \\
\hline Sky & - & - & 82 & - & 151 & 100 \\
\hline Tree (Left) & 192 & 82 & - & - & 94 & - \\
\hline Tree (Right) & 89 & - & - & - & - & 74 \\
\hline Build (Left) & - & 151 & 94 & - & - & - \\
\hline Build (Right) & 29 & 100 & - & 74 & - & - \\
\hline $\begin{array}{l}\overline{\mathcal{I}}, \mathcal{C}(\mathcal{Y}) \rightarrow \\
\downarrow\left\{S_{\mathcal{c}}(\mathcal{I}, \mathcal{C}(\mathcal{Y}))\right\}\end{array}$ & Road & Sky & $\begin{array}{c}\text { Tree } \\
\text { (Left or Right) }\end{array}$ & $\begin{array}{c}\text { Build } \\
\text { (Left or Right) }\end{array}$ & & \\
\hline$\overline{\text { Road }}$ & 1 & 1 & 0 & 0 & & \\
\hline Sky & 1 & 1 & 0 & 0 & & \\
\hline Tree (Left or Right) & 0 & 0 & 1 & 0 & & \\
\hline Build (Left or Right) & 0 & 0 & 0 & 1 & & \\
\hline
\end{tabular}

\subsection{Building image}

Figure $11 \mathrm{a}$ is the image to be interpreted using the knowledge acquired and tabulated in table 5. Observe that we have two interpretation labels (table 5) corresponding to the Tree, namely, Tree (left) and Tree (right), in our knowledge base, this to take care of the fact that tree cover occurs in two different shapes, having different gray level variation. Nevertheless, we interpret either of the labels as Tree in our simulations and hence have a single legend (figure 11). A similar observation is true for the interpretation label build. Figure $1 \mathrm{lb}$ is the wavelet-transformed image, figure $11 \mathrm{c}$ is the output of the $k$-means segmentation algorithm and figure $11 \mathrm{f}$ is the resultant image obtained after refining the $k$-means segmented image using the difference image information present in figure 11b. Figure 11d depicts segmentation and figure $11 \mathrm{e}$ interpretation of the scene at an intermediate stage. Figure $11 \mathrm{~g}$ gives the final interpreted image. In this example, the interpretation merges segments of sky by assigning them labels corresponding to sky. In fact, as seen from figure $11 \mathrm{~g}$, there are three different segments from figure $11 \mathrm{f}$ which have been correctly assigned the same label, sky.

\subsection{Computer images}

Figures $12 \mathrm{a}$ and $13 \mathrm{a}$ are images captured in the laboratory using the Pulnix CCD camera with zoom. The interpretations that we are looking for are: (i) background, (ii) screen, (iii) screen-frame, (iv) keyboard, (v) shoebox and (vi) table-top. Figures $12 \mathrm{~b}$ and $13 \mathrm{~b}$ are the wavelet-transformed images of figures $12 \mathrm{a}$ and $13 \mathrm{a}$ respectively. Figures $12 \mathrm{c}$ and $13 \mathrm{c}$ 


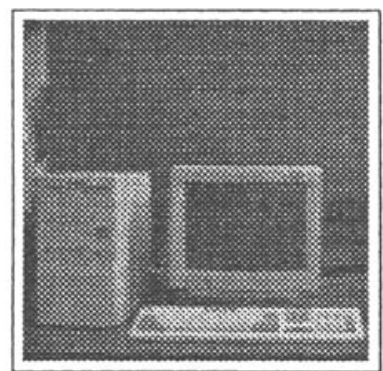

(a)

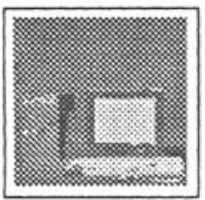

(c)

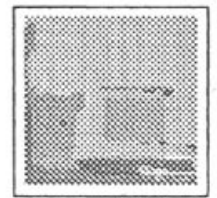

(d)

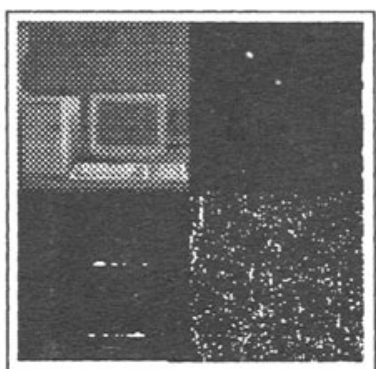

(b)

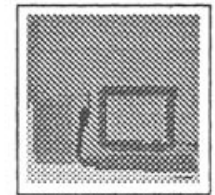

(e)

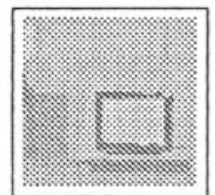

(f)

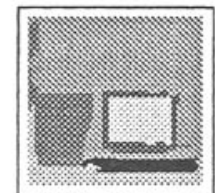

(g)

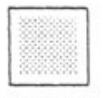

screen

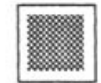

screen-frame
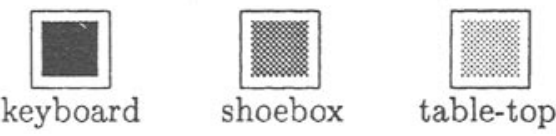

Figure 12. (a) Original scene image of size $256 \times 256$; (b) wavelet transformed image; (c) initial segmentation (after $k$-means clustering) of size $128 \times 128$; (d) segmentation at an intermediate stage: (e) interpretation at an intermediate stage; (f) final segmentation of size $128 \times 128$; $(\mathrm{g})$ final interpretation, and the interpretation legends corresponding to $(\mathrm{g})$.

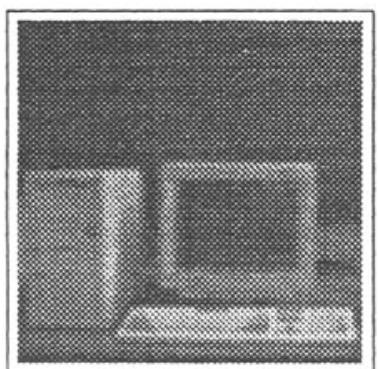

(a)

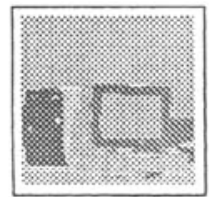

(c)

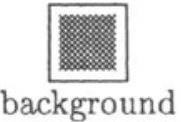

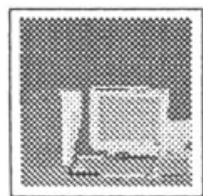

(d)

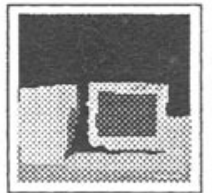

(e)

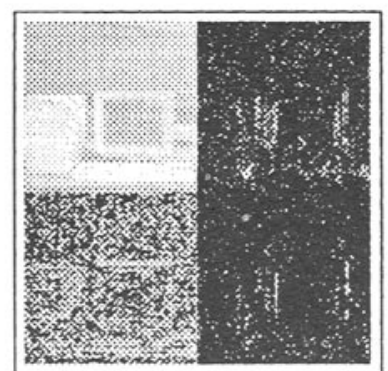

(b)

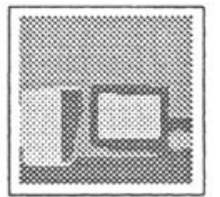

(f)

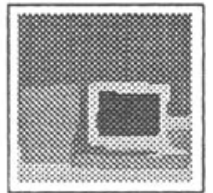

(g)

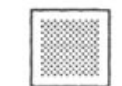

table-top

Figure 13. (a) Original scene image of size $256 \times 256$ : (b) wavelet transformed image; (c) initial segmentation (after $k$-means clustering) of size $128 \times 128$; (d) segmentation at an intermediate stage; (e) interpretation at an intermediate stage; (f) final segmentation of size $128 \times 128$; $(\mathrm{g})$ final interpretation, and the interpretation legends corresponding to $(\mathrm{g})$. 
Table 6. Knowledge base used for the indoor computer images (figures 12 and 13).

\begin{tabular}{lcccccc}
\hline Label & A & G & P & $m_{x}$ & $m_{y}$ & V \\
\hline Background & 8334 & 36 & 785 & 66 & 36 & 6.749894 \\
Screen & 1510 & 38 & 158 & 80 & 75 & 2.292660 \\
Screen-frame & 1537 & 39 & 439 & 86 & 78 & 3.056080 \\
Keyboard & 1067 & 74 & 203 & 82 & 111 & 34.836517 \\
Shoebox & 2401 & 35 & 212 & 21 & 82 & 7.556810 \\
Table-top & 1529 & 77 & 323 & 56 & 121 & 30.613126 \\
\hline & & & & & & \\
\hline Common & Background & Screen & Screen- & Keyboard & Shoebox & Table-top \\
perimeter $(C P)$ & & & frame & & & \\
\hline Background & - & - & 426 & 432 & 422 & - \\
Screen & - & - & & 296 & - & - \\
Screen-frame & 426 & 296 & - & - & - & - \\
Keyboard & 432 & - & - & - & - & 180 \\
Shoebox & 422 & - & - & - & - & 79 \\
Table-top & - & - & - & 180 & 79 & - \\
\hline & & & & & & \\
\hline $\mathcal{I}, \quad \mathcal{C}(\mathcal{Y}) \rightarrow$ & Background & Screen & Screen- & Keyboard & Shoebox & Table-top \\
$\downarrow\left(S_{c}(\mathcal{I}, \mathcal{C}(\mathcal{Y}))\right)$ & & & frame & & & \\
\hline Background & 1 & 1 & 0 & 0 & 0 & 1 \\
Screen & 1 & 1 & 1 & 0 & 1 & 1 \\
Screen-Frame & 0 & 0 & 1 & 1 & 1 & 1 \\
Keyboard & 0 & 1 & 1 & 1 & 1 & 0 \\
Shoebox & 0 & 1 & 1 & 1 & 1 & 0 \\
Table-top & 1 & 1 & 1 & 0 & 0 & 1 \\
\hline & & & & & &
\end{tabular}

are the output of the $k$-means segmentation algorithm after refinement of figures 12a and 13a respectively. Figures $12 \mathrm{f}$ and $13 \mathrm{f}$ are the final segmentation, while figures $12 \mathrm{~g}$ and $13 \mathrm{~g}$ are the finally interpreted images. An intermediate stage in the joint segmentation and interpretation scheme is shown in figures $12 \mathrm{~d}$ and $13 \mathrm{~d}$ (segmentation), and figures $12 \mathrm{e}$ and $13 \mathrm{e}$ (interpretation). The knowledge base that was used for the purpose of interpretation is shown in table 6.

As seen earlier, interpretation helps in refining segmentation and hence producing better segmentation. Figure $12 \mathrm{f}$ shows the shoebox as three segments, while after interpretation the three segments of the shoebox get labelled as shoebox (see figure 12g) and hence form a single segment. The spot above the shoebox looks like a rectangular strip and hence gets labelled as a shoebox, though it is because of a shadow formed by a black cloth kept behind the computer while capturing the image. A similar situation is seen in figure $13 \mathrm{~g}$ where the background (above the keyboard and to the right of the monitor screen-frame) gets labelled as screen because of the shape which looks more like a screen. But for these, the labelling is correct in both the examples.

The three sets of experimental results show that the proposed framework of modular integration and multiresolution produces results which can only be better than that produced if there were to be no integration between the modules (just a feed-forward interaction). The multiresolution aspect of the proposed framework not only helps in refining the segmented image but we also find that we need not interpret the image at the finest resolution namely $256 \times 256$; it is sufficient to stop interpretation at one level coarser, namely at $128 \times 128$, 
without affecting the interpretation results. This helps in reducing computational cost. Typically, the time taken on an IBM Pentium, running Linux OS in a multiuser environment for the entire process of image interpretation using the proposed joint segmentation and image interpretation is $\approx 54$ seconds.

\section{Conclusion}

Motivated by the functioning of the human visual system, we have proposed a modular integration and multiresolution framework for solving any vision task in general. We have validated the proposed framework by applying it to the high-level vision task of image interpretation. The fact that the given vision task can be best solved by first dividing the task into smaller subtasks and then synergetically integrating them is demonstrated by the simulation results where it is observed that the results obtained using the proposed scheme can only be better than those obtained when the modules have only feed-forward interaction. Also, due to the use of multiresolution in the proposed framework, the interpretation process is computationally fast ( $\approx 54$ seconds). Though, we have used images, which are of size $2^{k} \times 2^{k}$ for our simulations, this is not a restriction on the algorithm. We could use images of any arbitrary size, the construction of the pyramid to be used in the joint segmentation and interpretation scheme would then follow details given by Peleg et al (1987) and Jolion \& Rosenfeld (1994).

\section{References}

Binford T 1982 Survey of model based image analysis systems. Int. J. Robotics Res. 1: 587-633 Bozma H I, Duncan J S 1994 A game-theoretic approach to integration of modules. IEEE Trans. Pattern Anal. Machine Intell. 16: 1074-1086

Chu C C, Aggarwal J K 1992 Image interpretation using multiple sensing modalities. IEEE Trans. Pattern Anal. Machine Intell. 14: 840-847

Clark J J, Yullie A L 1990 Data fusion for sensory information processing systems (Norwell, MA: Kluwer)

Clement V, Thonnat M 1993 A knowledge-based approach to integration of image processing procedures. CVGIP: Image Understanding 55: 166-184

Gamble E B, Geiger D, Poggio T A, Weinshall D 1989 Integration of vision modules and labelling of surface discontinuities. IEEE Trans. Syst., Man Cybern. 19: 1576-1581

Geman S, Geman D 1984 Stochastic relaxation, Gibbs distribution, and Bayesian restoration of images. IEEE Trans. Pattern Anal. Machine Intell. 6: 721-741

Jeng 1992 Subsampling of Markov random fields. J. Visual Commun. Image Representation 3: $225-229$

Jolion J M, Rosenfeld A 1994 A pyramid framework for early vision (Norwell, MA: Kluwer)

Kim I Y, Yang H S 1993 Efficient image labelling based on Markov random field and error backpropagation network. Pattern Recogn. 26: 1695-1707

Kim I Y, Yang H S 1995 An integrated approach for scene understanding based on Markov random field. Pattern Recogn. 28: 1887-1897

Kumar V P, Desai U B 1996 Image interpretation using Bayesian networks. IEEE Trans. Pattern Anal. Machine Intell. 18: 74-77 
Lakshmanan S, Derin H 1993 Gaussian MRF at multiple resolutions. In Markov Random Fields - theory and applications (eds) R Chellappa, A Jain (San Diego: Academic Press) ch. 6, pp. $131-158$

Li S Z 1995 MRF modeling in computer vision (Tokyo: Springer-Verlag)

Mallat S G 1989 Multifrequency channel decompositions of images and wavelet models. IEEE Trans. Acoust. Speech Signal Process. 37: 2091-2110

Modestino J A, Zhang J 1992 A Markov random field model based approach to image interpretation. IEEE Trans. Pattern Anal. Machine Intell. 14: 606-615

Nagao M, Matsuyama T 1980 A structural analysis of complex aerial photographs (New York: Plenum)

Nanda P K, Sunil Kumar K, Gokhale S, Desai U B 1995 A multiresolution approach to color image resioration and parameter estimation using homotopy continuation method. In Proceedings Int. Conf. on Image Processing, Washington, DC

Ohta Y 1985 Knowledge based interpretation of outdoor natural color scenes (Boston: Pitman)

Peleg S, Federbusch O, Hummel R 1987 Custom made pyramids. In Parallel computer vision (ed.) L Uhr (Boston: Academic Press) pp. 125-146

Perez P, Heitz F 1994 Restriction of a MRF on a graph and multiresolution image analysis. Tech. Rep. RR 2170, INRIA, Sophia-Antipolis Cedex, France

Puliti P, Tascini G 1993 Knowledge-based approach to image interpretation. Image Vision Comput. 11: $122-128$

Rosenfeld A 1984 Multiresolution image processing and analysis (Berlin: Springer-Verlag)

Schalkoff R J 1989 Digital image processing and computer vision (Singapore: John Wiley and Sons)

Sonka M, Tadikonda S K, Collins S M 1993 Genetic algorithms in hypothesize-and-verify image interpretation. Proc. SPIE - Sensor Fusion VI, pp. 236-247

Sunil Kumar K, Desai U B 1994 New algorithms for 3D surface description from binocular stereo using integeration. J. Franklin Inst. B331: 531-554

Sunil Kumar K, Desai U B 1996 Joint segmentation and image interpretation. Tech. Rep. SPANN.96.2, Indian Institute of Technology - Bombay (http://144.16.100.100/nil/doc/ jseginter/tr.html).

Tenenbaum J M, Barrow H G 1977 Experiments in interpretation-guided segmentation. Artif. Intell. 8: 241-274

Van Essen D C, Anderson C H, Felleman D J 1992 Information processing in the primate visual system: an integrated systems perspective. Science 255: 419-423 